\title{
Resurgence of Urgent-Start Peritoneal Dialysis in COVID-19 and Its Application to Advanced Heart Failure
}

\author{
Amir Ahmad Nassiri $^{a}$ Claudio Ronco ${ }^{b, c}$ Amir Kazory ${ }^{d}$ \\ aDepartment of Nephrology and Dialysis, Shahid Beheshti University of Medical Sciences, Tehran, Iran; ${ }^{b}$ Department \\ of Nephrology, San Bortolo Hospital and International Renal Research Institute of Vicenza (IRRIV), Vicenza, Italy; \\ 'Department of Medicine, University of Padova, Padova, Italy; ${ }^{\mathrm{d} D i v i s i o n}$ of Nephrology, Hypertension, and Renal \\ Transplantation, University of Florida, Gainesville, FL, USA
}

Unforeseen twists and turns are not unusual in medicine. The quest for the optimal management of a disease can end up serving another patient population, whether as a side benefit or as a full-fledged effect. A recent example is the advent of sodium-glucose cotransporter-2 inhibitors for diabetes, now becoming a pillar of therapy for patients with chronic kidney disease (CKD) or heart failure (HF), with or without diabetes. Herein, we provide an overview of a number of long-lasting barriers in the field of peritoneal dialysis (PD) that could be addressed owing to the efforts originally aimed at fighting the current pandemic. We then explain how this experience could conceivably result in improved management of patients with HF through a number of seemingly unrelated steps.

\section{Urgent-Start PD}

Despite recommendations of various professional societies around the world, a significant subset of patients with end-stage kidney disease (ESKD) do not receive preESKD education or access planning. For instance, $1 / 3$ of patients with incident ESKD were reported to have received little or no pre-ESKD care [1]. Added to this group
(C) 2021 The Author(s).

Published by S. Karger AG, Basel

This article is licensed under the Creative Commons AttributionNonCommercial-NoDerivatives 4.0 International License (CC BYNC-ND) (http://www.karger.com/Services/OpenAccessLicense). Usage and distribution for commercial purposes as well as any distribution of modified material requires written permission. are those who experience an unexpected acceleration in progression of their CKD not allowing them to have an appropriate functional dialysis access when they need to initiate maintenance renal replacement therapy (RRT). The great majority of such patients (sometimes referred to as "crash ESKD") are treated with urgent-start hemodialysis through a central venous catheter [1]. Urgentstart PD, typically referring to commencing PD within 2 weeks of catheter insertion, has been proposed as an alternative modality for these patients. The idea has been around for several years but it has largely remained underutilized as the evidence informing its practice has not been considered strong [2]. While there is a multitude of reasons impeding implementation of urgent-start PD (USPD), concerns about adequate familiarity of physicians and staff as well as lack of a robust infrastructure allowing such practice have been proposed as potential explanations [3].

\section{PD for Acute Kidney Injury}

Extracorporeal blood purification techniques such as continuous RRT are the most commonly used therapeutic modality for acute kidney injury (AKI) in developed coun- 


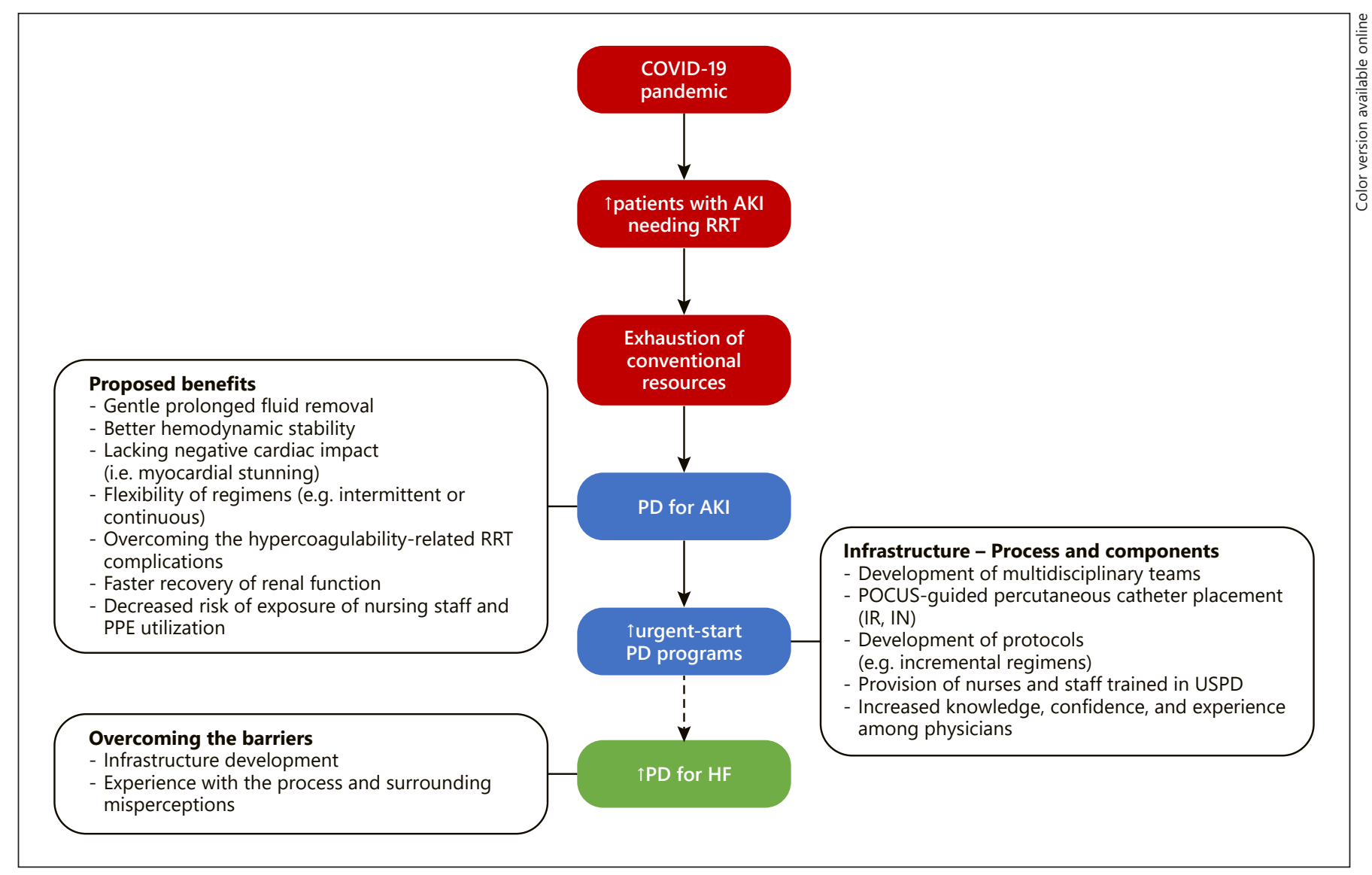

Fig. 1. Peritoneal dialysis: from COVID-19 to heart failure. During the pandemic, massive influx of critically ill patients with AKI has resulted in many centers around the world not having sufficient resources to offer acute hemodialysis or continuous extracorporeal RRTs. Consequently, we observe rapid development of USPD programs to manage these patients. This invaluable experience can be used as a model to expand the boundaries of PD therapy and

tries. PD represents an intriguing option for management of AKI in low-resource settings due to its lower cost. Currently available literature suggests similar outcomes for $\mathrm{PD}$ and conventional extracorporeal therapies in AKI [4]. Moreover, a number of advantages for PD in this setting have been proposed such as more rapid recovery of renal function and lack of exposure of blood to synthetic surfaces (Fig. 1). Nevertheless, PD use has been extremely limited in North America and Europe for a variety of reasons. Lack of familiarity of nephrologists with the use of PD in the acute setting and the misperception that $\mathrm{PD}$ portends inadequate clearance for these patients are among those [3, 4]. Similar to crash ESKD population, technical considerations also seem to contribute to this practice gap and the lack of uptake of PD for management of AKI [5]. enhance the care of patients with advanced HF. COVID-19, coronavirus disease of 2019; AKI, acute kidney injury; RRT, renal replacement therapy; PD, peritoneal dialysis; HF, heart failure; IR, interventional radiologist; IN, interventional nephrologist; POCUS, point-of-care ultrasound; USPD, urgent-start peritoneal dialysis; PPE, personal protective equipment.

\section{COVID-19 and PD}

The coronavirus disease of 2019 (COVID-19), caused by severe acute respiratory syndrome coronavirus 2 (SARS-CoV2), continues to spread around the world. AKI is one of the most serious complications of COVID-19 and a major determinant of resource utilization especially if RRT is needed. The unanticipated surge in the number of patients with AKI, limited pool of trained staff, scarce supplies of continuous RRT, and a higher rate of circuit clotting could quickly overwhelm the existing management options in this crisis and imposed rapid adaptation and creative problem-solving. As such, there has been a renewed interest in the use of PD for AKI. Indeed, an unprecedented number of urgent $\mathrm{PD}$ programs have 
been developed over the last few months in response to renal complications of COVID-19 pandemic $[6,7]$. Accumulating body of evidence has confirmed that USPD is an effective and safe alternative to conventional RRT modalities in the acute setting. It can indeed be the only option for those patients with recalcitrant clotting on continuous RRT or conventional hemodialysis. Moreover, we have learned how to quickly set up a collaborative urgent-start (or acute) PD program that includes appropriate patient selection, bedside PD catheter implantation, and implementation of customized PD regimens [8]. A number of practical considerations (e.g., training of interventional nephrologists or radiologists for point-of-care ultrasound- or fluoroscopy-guided catheter placement, development of PD protocols customized to the needs of these patients, increasing the capacity of the wards, and modification of the supply procurement and stocking methods) have been integrated into these efforts that are typically multidisciplinary $[7,8]$. While PD is not contraindicated in patients who need mechanical ventilation, lower initial dwell volumes might be preferred to monitor the impact of increasing intra-abdominal pressures on the ventilator mechanics, especially in those with rapidly expanding lung lesions. Although the long-term outcomes of COVID-19-associated AKI have yet to be delineated, AKI survivors often have incomplete recovery of kidney function at hospital discharge; with one-third of patients who received acute RRT having a persistent requirement for dialysis at the time of hospital discharge [9]. As such, a subset of patients treated with PD in the hospital can transition to this home-based therapy after discharge. Uncertainty exists whether SARS-CoV2 can be found in the PD effluent of the patients with active COVID-19, and whether it has the potential to replicate. Nevertheless, as the virus is present in other body fluids, it is recommended that precautions be taken when disposing of PD effluent.

\section{PD and HF}

Over the past 2 decades, PD has emerged as an effective and safe therapy for patients with advanced HF and refractory fluid overload. In this patient population, the course of the disease is typically complicated by frequent hospital admissions due to recurring episodes of acute decompensated HF and associated congestive manifestations despite high doses of diuretics. Peritoneal ultrafiltration, a home-based therapy, provides adequate fluid and sodium removal to prevent progressive accumulation of fluid in the outpatient setting that ultimately leads to admission of these patients [10]. In a subset of these patients with concomitant renal dysfunction, PD can also provide clearance of waste products. Indeed, flexibility of PD regimens allows for dynamic customization of this therapeutic modality to the clinical characteristics and needs of each patient [11]. Several studies have reported beneficial effects associated with the use of PD in patients with HF; these include reduction in hospital admissions, enhancement of cardiac function, and improvement in patients' quality of life. For example, a study by Courivaud et al. [12] reported a remarkable $90 \%$ reduction in the number of days of hospitalization after initiation of PD in patients suffering from advanced HF. Despite encouraging results and the steady increase in the number of patients with advanced HF, PD is not yet widely used for this patient population for a number of reasons. Interestingly, similar to those with crash ESKD, lack of an appropriate infrastructure seems to be a key barrier hindering this approach. Moreover, a unique challenge in the care of these patients is that due to their heavy cardiovascular morbidity, they are typically poor surgical candidates for placement of a PD catheter by the time they reach the point that they need it [10].

\section{Closing the Loop}

COVID-19 has pushed us to our limits by challenging the conventional approaches and exhausting our resources. It has forced us to come out of our comfort zone and consider the pre-existing unconventional options that we had previously ignored or underutilized (e.g., PD therapy for AKI). More importantly, the pandemic proved that it is feasible to set up effective multidisciplinary acute or USPD programs with point-of-care ultrasound-guided PD catheter implantation. Two key barriers to wider use of PD in this setting (e.g., lack of appropriate infrastructure and experience with bedside catheter placement) that previously seemed insurmountable have successfully been handled by numerous large centers especially those in metropolitan areas where COVID-19 flares were lingering. Not only should we embrace and enhance these experiences beyond a simple COVID-19 necessity, but we can expand our field by applying those practices to broader nonrenal indications such as advanced HF that represents a common, costly, and deadly disease (Fig. 1). 


\section{Statement of Ethics}

Human and animal rights: this article does not contain any studies with human participants or animals performed by any of the authors.

\section{Conflict of Interest Statement}

A.K. has the following potential conflicts of interest: Baxter, Inc. (Cardiology Advisory Board and consultancy fee), CHF Solutions, Inc. (Medical Advisory Board and consultancy fee), and W.L.Gore Inc. (consultancy fee).

\section{Funding Sources}

The authors did not receive any funding.

\section{Author Contributions}

A.K. - drafted the initial version of the manuscript. C.R. - revised the manuscript critically for important intellectual content. A.A.N. - revised the manuscript critically for important intellectual content. All authors have read and approved the manuscript prior to final submission.

\section{References}

1 United States Renal Data System Annual Data Report (USRDS). Available from: https:// www.usrds.org/annual-data-report/currentadr/ Accessed 2020 Oct 15.

2 Tunbridge M, Cho Y, Johnson DW. Urgentstart peritoneal dialysis: is it ready for prime time? Curr Opin Nephrol Hypertens. 2019; 28(6):631-40.

3 Berns JS. A survey-based evaluation of selfperceived competency after nephrology fellowship training. Clin J Am Soc Nephrol. 2010;5(3):490-6.

4 Chionh CY, Soni SS, Finkelstein FO, Ronco C, Cruz DN. Use of peritoneal dialysis in AKI: a systematic review. Clin J Am Soc Nephrol. 2013;8(10):1649-60.
5 Ponce D, Gobo-Oliveira M, Balbi AL. Peritoneal dialysis treatment modality option in acute kidney injury. Blood Purif. 2017;43(13):173-8.

6 Parapiboon W, Ponce D, Cullis B. Acute peritoneal dialysis in COVID-19. Perit Dial Int. 2020;40(4):359-62.

7 Brown EA, Perl J. Increasing peritoneal dialysis use in response to the COVID-19 pandemic: will it go viral? J Am Soc Nephrol. 2020; 31(9):1928-30.

8 Sourial MY, Sourial MH, Dalsan R, Graham J, Ross M, Chen W, et al. Urgent peritoneal dialysis in patients with COVID-19 and acute kidney injury: a single-center experience in a time of crisis in the united states. Am J Kidney Dis. 2020;76(3):401-6.
9 Wald R, Bagshaw SM. COVID-19-associated acute kidney injury: learning from the first wave. J Am Soc Nephrol. 2020;32.

10 Kazory A, Bargman JM. Defining the role of peritoneal dialysis in management of congestive heart failure. Expert Rev Cardiovasc Ther. 2019;17(7):533-43.

11 Kazory A, Koratala A, Ronco C. Customization of peritoneal dialysis in cardiorenal syndrome by optimization of sodium extraction. Cardiorenal Med. 2019;9(2):117-24.

12 Courivaud C, Kazory A, Crépin T, Azar R, Bresson-Vautrin C, Chalopin JM, et al. Peritoneal dialysis reduces the number of hospitalization days in heart failure patients refractory to diuretics. Perit Dial Int. 2014;34(1): $100-8$. 